Original Article

\title{
Effects of Nordic walking on physical functions and depression in frail people aged 70 years and above
}

\author{
Han Suk Lee, PhD, PT ${ }^{1)^{*}}$, Jeung Hun Park, $\mathrm{PT}^{2)}$ \\ 1) Department of Physical Therapy, Faculty of Health Science, Eulji University: 553 San Sung Ro, \\ Sujeong-gu, Seongnam, Gyeonggi-do 461-815, Republic of Korea \\ 2) Department of Physical Therapy, Sujeong Jungang Senior Welfare Center, Republic of Korea
}

\begin{abstract}
Purpose] This study investigated the effects of Nordic walking on physical functions and depression in frail people aged 70 years and above. [Subjects] Twenty frail elderly individuals $\geq 70$ years old were assigned to either a Nordic walking group $(n=8)$ or general exercise group $(n=10)$. [Methods] The duration of intervention was equal in both groups (3 sessions/week for 12 weeks, $60 \mathrm{~min} / \mathrm{session}$ ). Physical function (balance, upper extremity strength, lower extremity strength, weakness) and depression were examined before and after the interventions. [Results] With the exception of upper extremity muscle strength, lower extremity strength, weakness, balance, and depression after Nordic walking demonstrated statistically significant improvement. However, in the general exercise group, only balance demonstrated a statistically significant improvement after the intervention. There were significant differences in the changes in lower extremity muscle strength, weakness and depression between the groups. [Conclusion] In conclusion, Nordic walking was more effective than general exercise. Therefore, we suggest that Nordic walking may be an attractive option for significant functional improvement in frail people over 70 years old.

Key words: Nordic walking, General exercise, Frail people
\end{abstract}

(This article was submitted Mar. 16, 2015, and was accepted Apr. 24, 2015)

\section{INTRODUCTION}

There are many possible causes of frailty including physical, social, and emotional factors arising from decreased physical activity, sensational hypofunction, physical symptoms or illness, and social isolation. As frailty increases the risk of accidents, frail older adults require greater levels of supervision during exercise ${ }^{1,2)}$.

Most programs for frail older adults are performed at homes or facilities to ensure safety ${ }^{3-7)}$. However, such programs will not yield positive emotional results if the participants are kept in a smaller space for exercise, although they are safe. This is because as the elderly advance further in age, they tend to live in smaller areas. Therefore, giving participants a chance to escape their routine and perform exercise in a larger space may provide positive emotional results.

Despite the positive effects of exercise, the rate of regular practical exercise performed by Korean adults including frail older adults is as low as $9.9-22.6 \%^{8)}$. In order to increase the rate of practical exercise, programs must be constructed with no limitation of facilities, space, or economic constraints ${ }^{9)}$.

*Corresponding author. Han Suk Lee (E-mail: leehansuk21@ hanmail.net)

(C2015 The Society of Physical Therapy Science. Published by IPEC Inc. This is an open-access article distributed under the terms of the Creative Commons Attribution Non-Commercial No Derivatives (by-ncnd) License $<$ http://creativecommons.org/licenses/by-nc-nd/3.0/> .
Nordic walking is an appropriate exercise activity that can be done easily anywhere and anytime. It can not only increase the rate of exercise ${ }^{10)}$ but also provide physical and psychological benefits to elderly people who live in small spaces by allowing them to exercise outdoors. Nordic walking is performed using poles intended to be swung forward and backward with the upper body, which may improve elderly individuals' upper-body muscle strength ${ }^{11)}$ and exercise the lower body while placing less force on the knee ${ }^{12)}$. Furthermore, outdoor activities improve not only physical activity but also depression ${ }^{13)}$.

Almost all studies on Nordic walking have focused on the cardiovascular system and involved participants with Parkinson's disease or healthy adults; studies on frail older adults with various underlying health issues are lacking. Furthermore, no studies have involved subjects over the age of 70, a time when activity levels sharply decrease.

Accordingly, we undertook the present study with the aim of assessing whether Nordic walking is an appropriate exercise for elderly subjects aged $\geq 70$ years and its effect on physical improvement and depression.

\section{SUBJECTS AND METHODS}

This study targeted frail older adults living in city $\mathrm{S}$ and was carried out over 12 weeks from August to December 2014. The 20 elderly individuals $\geq 70$ years old selected to participate in this study lived in city S, Gyeonggi-do, South Korea, visited the S Senior Welfare Center, and were randomly assigned to the Nordic walking group or general 
exercise group. The inclusion criteria were as follows: ability to understand and agree with the purpose of this study, ability to communicate and demonstrated normal cognition (MMSEK [Mini Mental State Examination-Korea]: a score $>24$ ), and more than 2 points based on the criteria for frailty in older adults. Prior to participation, all participants were informed about the study's aims and procedures and signed informed consent forms. This study conformed to the ethical principles of the Declaration of Helsinki. The general characteristics of the subjects are shown in Table 1.

Pre- and post-intervention assessments were performed in the Nordic walking and general exercise groups. The assessments included measurements of state of weakness, physical function, and depression. The Korean Health Compliance Frailty Assessment Tool for the Elderly, developed by Yoon ${ }^{14)}$, was used to assess frailty. It includes a total of 8 items. Scores below 2 are normal; a score $\geq 2$ indicates weakness, and a score $\geq 5$ indicates severe weakness.

The physical function assessment tool was used to measure physical function in the elderly ${ }^{15}$ ). The items consisted of balance, lower extremity muscle strength, and upper extremity muscle strength. The balance test involved standing on one leg with the eyes open. The time that a single-leg stance could be maintained was measured, with the time determined by when the raised leg touched the ground or when the foot moved from the origin. Lower extremity muscle strength was assessed by measuring the number of times the subject could stand up from a 46-cm-high chair, which had no armrests, with their arms crossed and hands on their shoulders before their hands fell from their positions. Upper arm muscle strength was assessed by measuring the number of times the subject was able to lift a 2-kg dumbbell above elbow level in 30 seconds.

The Korean version of the Short Geriatric Depression Scale developed in the research of $\mathrm{Bae}^{16)}$ was used to assess depression. It includes a total of 15 items, each requiring a "yes" or "no" response. Scores of 0-7 points are considered normal, and scores of 8-15 are indicative of depression.

For the Nordic walking group, Nordic walking was conducted three times per week for 12 weeks. Considering the characteristics of frail older adults, walking practice was performed twice per week using a treadmill indoors and once per week outdoors. During the first 4 weeks, subjects walked at a medium tempo with medium strength in a flat park, and during the next 4 weeks, they walked at the same tempo and strength but in a hilly park. During the final 4 weeks, they walked around a mountain with broad, low hills at a medium tempo and medium strength. The control group was asked to perform a selected general exercise program indoors for 1 hour three times per week. The general exercise program consisted of whole-body stretching and simple muscle strength exercises, and it was an exercise program used for the purpose of improving flexibility and muscle strength in the elderly.

The general characteristics of the test and control groups were analyzed by using their means and standard deviations, and the Shapiro-Wilk test was used to confirm that values of dependent variables were normally distributed. According to the results of the Shapiro-Wilk test, the Mann-Whitney $\mathrm{U}$ test and independent t-test were used for comparisons of
Table 1. The general characteristics of the subjects

\begin{tabular}{lcc}
\hline & $\begin{array}{c}\text { General exercise } \\
(\mathrm{n}=10)\end{array}$ & $\begin{array}{c}\text { Nordic walking } \\
(\mathrm{n}=8)\end{array}$ \\
\hline Age (years) & $73.9(1.4)$ & $75.2(3.9)$ \\
Height $(\mathrm{cm})$ & $148.6(4.3)$ & $151.8(6.3)$ \\
Weight $(\mathrm{kg})$ & $50.2(6.0)$ & $56.0(5.5)$ \\
\hline
\end{tabular}

Table 2. Effects of the Nordic walking program

\begin{tabular}{lcc}
\hline Items (unit) & $\begin{array}{c}\text { Before } \\
\text { Nordic } \\
\text { walking }\end{array}$ & $\begin{array}{c}\text { After } \\
\text { Nordic } \\
\text { walking }\end{array}$ \\
\hline Balance (sec) & $22.38(18.8)$ & $52.38(28.33)^{*}$ \\
Lower extremity strength (number) & $18.88(4.08)$ & $23.75(3.28)^{*}$ \\
Upper extremity strength (number) & $29.75(5.36)$ & $31.13(3.94)$ \\
Weakness (score) & $3.19(1.06)$ & $2.13(1.12)^{*}$ \\
Depression (score) & $2.25(2.18)$ & $1.0(1.41)^{*}$ \\
\hline
\end{tabular}

$* \mathrm{p}<0.05$

weakness and all other variables, respectively, between the two groups. In order to determine the effect of the program, the paired t-test was used to compare pre-intervention and post-intervention values. Statistical analysis was performed using SPSS Statistics 18.0, and significance was accepted for values of $\mathrm{p}<0.05$.

\section{RESULTS}

The scores for balance, lower extremity muscle strength, weakness, and depression all showed improvement in those who participated in the Nordic walking program. With the exception of upper extremity muscle strength, all items demonstrated statistically significant improvement (Table 2).

In the general exercise group, only balance demonstrated a statistically significant improvement. Scores for lower extremity muscle strength, upper extremity muscle strength, weakness, and depression showed no significant difference between before and after the general exercise program (Table 3).

Looking at the change in balance, the Nordic walking group increased by 1.37 but the general exercise group decreased by -0.90 , and there was no significant difference between groups. Lower extremity muscle strength increased in the Nordic group, with the number of stands increasing by 4.87 , but it decreased in the general exercise group, with the number of stands decreasing by 0.20 , and the difference in change between the groups was statistically significant. In the case of upper extremity muscle strength, the Nordic walking group showed improvement, with the number of lifts increasing by 1.37 , but the general exercise group showed a decrease, with the number of lifts decreasing by 0.9. However, the difference between the two groups was not statistically significant. Weakness improved slightly in the Nordic walking group, with the score decreasing by 1.06, but showed little improvement in the general exercise group, with the score decreasing by 0.10 . The change in weakness 
Table 3. Effects of the general exercise program

\begin{tabular}{lcc}
\hline Items (unit) & $\begin{array}{c}\text { Before } \\
\text { exercise }\end{array}$ & $\begin{array}{c}\text { After } \\
\text { exercise }\end{array}$ \\
\hline Balance (sec) & $25.7(30.5)$ & $38(28.5)^{*}$ \\
Lower extremity strength (number) & $19.5(3.0)$ & $19.7(3.4)$ \\
Upper extremity strength (number) & $29.4(4.7)$ & $28.5(4.4)$ \\
Weakness (score) & $3.5(1.7)$ & $3.4(1.8)$ \\
Depression (score) & $3.5(2.7)$ & $3.5(2.7)$ \\
\hline$* p<0.05$ & &
\end{tabular}

$* \mathrm{p}<0.05$

Table 4. Comparison of the change between the Nordic walking program and General exercise program

\begin{tabular}{lcc}
\hline Items & $\begin{array}{c}\text { General } \\
\text { exercise }\end{array}$ & $\begin{array}{c}\text { Nordic } \\
\text { walking }\end{array}$ \\
\hline Balance & $-0.9(1.28)$ & $1.37(4.68)$ \\
Lower extremity strength & $0.2(1.31)$ & $4.87(3.27)^{*}$ \\
Upper extremity strength & $-0.9(1.28)$ & $1.37(4.68)$ \\
Weakness & $-0.1(0.31)$ & $-1.06(0.94)^{*}$ \\
Depression & $0.0(0.0)$ & $-1.25(1.28)^{*}$ \\
\hline
\end{tabular}

$* \mathrm{p}<0.05$

in both groups was statistically significant. The score for depression after Nordic walking improved by 1.25 , showing improvement compared with the general exercise group's score of 0 , and the difference between groups was statistically significant (Table 4).

\section{DISCUSSION}

As society is increasingly aging today, the need for special consideration for frail adults over 70 years old is increasing. Exercises for such adults must be easy to follow and simple and should have a limited economic burden if the goal is to maintain continued participation in the exercises. Nordic walking is one exercise that may be recommended on the basis of satisfying these criteria. As there is limited research on Nordic walking in frail older adults, this study investigated whether a Nordic walking program could improve physical function and depression in frail adults over 70 years old.

Nordic walking has been reported to result in increased walking distance ${ }^{17}$, rectus femoris activity ${ }^{12)}$, and muscle strength ${ }^{11,18)}$ compared with a standard home exercise group. In other words, the improvement in lower extremity muscle strength was very good. Lower extremity muscle strength improved, and compared with the general exercise group, the level of improvement was also very high in the present study. This increase in muscle strength may have been the result of using the walking poles, allowing adults with possible degeneration of the knees to place less of a burden on them while performing the walking exercise naturally.

However, Kukkonen-Harjula et al. ${ }^{19)}$ claimed that walking with a pole resulted in less improvement in fitness and oneleg squat strength than fast walking. Differences in subjects' ages and the intensity of the exercise are likely reasons for such conflicting findings. The study by Kukkonen-Harjula et al. ${ }^{19)}$ targeted obese women in their $50 \mathrm{~s}$ and $60 \mathrm{~s}$, while the present study focused on frail adults aged $\geq 70$ years. Furthermore, as the purpose of the previous study was to reduce obesity, strenuous exercises such as brisk walking were chosen, which have been proved superior to Nordic walking in improving obesity and muscle strength. As the present study was not directed at reducing obesity but rather was directed at improving the physical function of frail older adults, the intensity of the exercises was lower than in the previous study.

As the elderly are at greater risk of falls, exercises aimed at strengthening muscles, Tai chi, and general walking have been recommended to increase balance. However, there is a difference in the effect of balance programs conducted in the elderly due to the structure of exercises. Howe et al. ${ }^{20)}$ reported that strength exercise increased the balance of older people but that general walking activity did not significantly increase the scores for the Timed Up and Go test, singleleg stance with eyes open, or single-leg stance with eyes closed or the self-paced gait speed among older people. Giné-Garriga et al. ${ }^{21)}$ found that exercise has some benefits in frail older people, although uncertainty remains with regard to which exercise characteristics (type, frequency, intensity, duration, setting, combinations) are most effective. Furthermore, no consistent effect was observed on balance or functional mobility.

The findings of a meta-analysis by Chou et al. ${ }^{22}$, which involved various types of exercise interventions, also demonstrated the beneficial effects of exercise on frail older adults. However, the most appropriate exercise program for balance (reflected by Timed Up and Go test performance) has yet to be determined, because no difference was found between various types of exercise interventions. Although balance improved in both the Nordic walking and general exercise group, as seen in previous research, the present study detected no difference in balance improvement between the two exercise groups. Therefore, we cannot recommend Nordic walking as a superior exercise for balance improvement in frail adults over 70 years old. Balance improvement can be affected by various factors such as the nervous system, musculoskeletal system, and the environment, and therefore it may be difficult for one exercise program to demonstrate significant improvement over other forms of exercise.

Additionally, in the present study, exercise was performed only once outdoors and twice indoors each week, which could be a factor reducing the degree of improvement shown in balance. Consequently, performance of Nordic walking outdoors more than once per week is needed to assess its maximal effects on balance and other variables.

According to studies by Song et al. ${ }^{11)}$ and Shim et al. ${ }^{23)}$, Nordic walking was more effective than general walking for improving upper extremity muscle strength in the elderly. However, in the present study, the improvement in upper extremity muscle strength was not statistically significant. This conflicting result may be the result of the different ages of subjects between the studies, since the previous study assessed elderly subjects or healthy subjects in their twenties but not specifically frail individuals aged over 70 years. In the case of frail adults aged over 70 years, once their muscle strength has declined substantially with age, the actions of 
holding the Nordic walking poles and upper extremity rotation may be insufficient to improve upper extremity muscle strength. Therefore, additional research is needed to identify positions in Nordic walking that can improve upper extremity muscle strength and add them to programs targeting people over 70.

Suija et al. ${ }^{13)}$ reported that regular Nordic walking improved the mood and physical fitness of depressed patients. Consistent with that study, the present results indicated an improvement in depression. This finding indicates that outdoor exercise has a greater effect on psychological stability than indoor exercise. The formation of vitamin D during outdoor exercise may be involved in improving depression, and conversations with other participants in a natural setting, in combination with the exercise, may help to maximize the psychological effect. Therefore, outdoor exercise programs involving group activity are more effective for improving depression.

The main limitation of this study was the small sample size available for analysis, owing to the number of patients who withdrew. The most common reason for high withdrawal rates in elderly subjects is their preference not to participate in community programs. Another limitation was the frequency of outdoor Nordic walking, which was once per week. We decided on this frequency of outdoor Nordic walking out of consideration for the conditions of the paricipants, since it was their first time participating in the activity. Future studies should ideally have a larger sample size and increased frequency of outdoor Nordic walking.

As a result of participating in a Nordic walking program for 12 weeks, frail adults over the age of 70 years demonstrated improvements in balance, lower extremity muscle strength, weakness, and depression. Overall, Nordic walking was more effective than general exercise. Therefore, we suggest that Nordic walking may be an attractive option for significant functional improvement in frail people over 70 years old.

\section{REFERENCES}

1) Bergman H, Ferrucci L, Guralnik J, et al.: Frailty: an emerging research and clinical paradigm - issues and controversies. J Gerontol A Biol Sci Med Sci, 2007, 62: 731-737. [Medline] [CrossRef]

2) Choi KW, Lee IS: The concept of frailty: a review of the literature. Korean J Rehabil Nurs, 2008, 11: 67-73.

3) Yoo JS, Jeon MY, Kim CG: [Effects of a fall prevention program on falls in frail elders living at home in rural communities]. J Korean Acad Nurs, 2013, 43: 613-625. [Medline] [CrossRef]

4) Kim YJ, Ha JY: The effects of visiting exercise program and telecoaching for physical activity promotion on physical fitness and quality of life in the frail elderly. Korean J Adult Nurs, 2011, 23: 198-207.
5) Sun WD, Lee SH, Park JS, et al.: Analysis of the effects of muscle strength exercise on physical function and quality of life in the frail elderly. J Korean Soc Health Educ Promot, 2008, 25: 39-53.

6) Nakagawa $\mathrm{K}$, Inomata $\mathrm{N}$, Konno $\mathrm{Y}$, et al.: The characteristic of a simple exercise program under the instruction of physiotherapists - for general elderly people and frail elderly people. J Phys Ther Sci, 2008, 20: 197-203.

7) Kawagoe M, Kajiya S, Mizushima, et al.: Effect of continuous home-visit rehabilitation on functioning of discharged frail elderly. J Phys Ther Sci, 2009, 21: 343-348. [CrossRef]

8) Park YI, Lee KY, Kim TI, et al.: The effects of exercise in the frail elderly. J Korean Acad Community Health Nurs, 2012, 23: 91-101. [CrossRef]

9) Burbank PM, Padula CA, Nigg CR: Changing health behaviors of older adults. J Gerontol Nurs, 2000, 26: 26-33, quiz 52-53. [Medline] [CrossRef]

10) Tschentscher M, Niederseer D, Niebauer J: Health benefits of Nordic walking: a systematic review. Am J Prev Med, 2013, 44: 76-84. [Medline] [CrossRef]

11) Song MS, Yoo $\mathrm{YK}$, Choi $\mathrm{CH}$, et al.: Effects of nordic walking on body composition, muscle strength, and lipid profile in elderly women. Asian Nurs Res Korean Soc Nurs Sci, 2013, 7: 1-7. [Medline] [CrossRef]

12) Yang SM: Influence of nordic walking upon degenerative knee osteoarthritis patients muscular activities and change. Korea J Sports Sci, 2011, 20: 1085-1095.

13) Suija K, Pechter U, Kalda R, et al.: Physical activity of depressed patients and their motivation to exercise: Nordic Walking in family practice. Int $\mathrm{J}$ Rehabil Res, 2009, 32: 132-138. [Medline] [CrossRef]

14) Yoon JL: The development of program support system to promote the health for community elderly to extend health life: The Korean Health Compliance Frailty Assessment Tool for the Elderly. Seoul, Ministry of Health \& Welfare, 2012.

15) Insurance National Health Service: Analysis of effect about health exercise service to promote for older people health. Seoul, 2007.

16) Bae JN, Cho MJ: Development of the Korean version of the Geriatric Depression Scale and its short form among elderly psychiatric patients. J Psychosom Res, 2004, 57: 297-305. [Medline] [CrossRef]

17) Spafford C, Oakley C, Beard JD: Randomized clinical trial comparing Nordic pole walking and a standard home exercise programme in patients with intermittent claudication. Br J Surg, 2014, 101: 760-767. [Medline] [CrossRef]

18) Kocur P, Deskur-Smielecka E, Wilk M, et al.: Effects of Nordic walking training on exercise capacity and fitness in men participating in early, short-term inpatient cardiac rehabilitation after an acute coronary syndrome-a controlled trial. Clin Rehabil, 2009, 23: 995-1004. [Medline] [CrossRef]

19) Kukkonen-Harjula K, Hiilloskorpi H, Mänttäri A, et al.: Self-guided brisk walking training with or without poles: a randomized-controlled trial in middle-aged women. Scand J Med Sci Sports, 2007, 17: 316-323. [Medline]

20) Howe TE, Rochester L, Neil F, et al.: Exercise for improving balance in older people (Review). The Cochrane Collaboration, 2011, 11: 1-205.

21) Giné-Garriga M, Roqué-Fíguls M, Coll-Planas L, et al.: Physical exercise interventions for improving performance-based measures of physical function in community-dwelling, frail older adults: a systematic review and meta-analysis. Arch Phys Med Rehabil, 2014, 95: 753-769.e3. [Medline] [CrossRef]

22) Chou CH, Hwang CL, Wu YT: Effect of exercise on physical function, daily living activities, and quality of life in the frail older adults: a metaanalysis. Arch Phys Med Rehabil, 2012, 93: 237-244. [Medline] [CrossRef]

23) Shim JM, Kwon HY, Kim HR, et al.: Comparison of the effects of walking with and without Nordic pole on upper extremity and lower extremity muscle activation. J Phys Ther Sci, 2013, 25: 1553-1556. [Medline] [CrossRef] 